\section{Facharztprüfung zur Erlangung des Facharzttitels FMH für Pneu- mologie und des Schwerpunkts Kinder- und Jugendmedizin, spez. pädiatrische Pneumologie}

Aufgrund des Weiterbildungsprogrammes, welches am 1. Januar 2001 bzw. 1. Juli 2001 in Kraft gesetzt wurde, ist die Teilnahme an der Facharztprüfung für diejenigen Kandidatinnen und Kandidaten Voraussetzung für die Erlangung des Facharzttitels FMH für Pneumologie und des Schwerpunkts Kinderund Jugendmedizin, spez. pädiatrische Pneumologie, welche ihre Weiterbildung bis am 30. Juni 1997 nicht abgeschlossen hatten. Es empfiehlt sich, die Facharztprüfung frühestens im letzten Jahr der reglementarischen Weiterbildung abzulegen (Art. 23 WBO).
Ort: Lungenliga Schweiz, Südbahnhofstrasse 14c, 3000 Bern 17

Datum: Donnerstag, 29. August 2002

Prüfungsgebühr: Die SGP erhebt eine Prüfungsgebühr von Fr. 650.-.

Anmeldung bis zum 31. März 2002 unter Beilage des Curriculums an eine der folgenden Adressen:

Erwachsenen-Pneumologie

PD Dr. Konrad Bloch,

UniversitätsSpital Zürich, Departement

Innere Medizin, Abteilung Pneumologie,

Rämistrasse 100, 8091 Zürich,

Tel. 0125538 28, Fax 0125544 51,

E-Mail: konrad.bloch@dim.usz.ch

Pädiatrische Pneumologie

PD Dr. Jürg Hammer,

Kinderspital Basel, Pneumologie,

Römergasse 8, 4005 Basel,

Tel. 06168565 65, Fax 06168550 59,

E-Mail: juerg.hammer@unibas.ch

Der Stoffkatalog kann bezogen werden beim Sekretariat der Schweizerischen Gesellschaft für Pneumologie (SGP), Südbahnhofstrasse 14c, Postfach 49, 3000 Bern 17, Tel. 03137820 30, Fax 03137820 31, E-Mail: k.lehmann@lung.ch
Examen de spécialiste en vue de l'obtention du titre de spécialiste FMH en pneumologie et de la formation approfondie en pneumologie à adjoindre au titre de spécialiste FMH en pédiatrie

Selon le programme de formation postgraduée entré en vigueur le $1^{\text {er }}$ janvier 2001 et $1^{\text {er }}$ juillet 2001, la participation à l'examen de spécialiste est une condition requise pour les candidats à l'obtention du titre de spécialiste FMH en pneumologie et pédiatrie, spéc. pneumologie pédiatrique qui n'avaient pas terminé leur formation postgraduée le 30 juin 1997. Il leur est recommandé de passer l'examen de spécialiste au plus tôt durant la dernière année de leur formation postgraduée réglementaire (art. 23 RFP).
Lieu: Ligue pulmonaire Suisse, Südbahnhofstrasse 14c, 3000 Berne 17

Date: Jeudi, 29 août 2002

Taxe d'examen: La SSP prélève une participation aux frais de Fr. 650.-.

Inscription: Envoyez votre inscription d'ici au 31 mars 2002, accompagné de votre curriculum, à l'une des adresses suivantes:

Pneumologie des adultes

PD Dr. Konrad Bloch,

UniversitätsSpital Zürich, Departement

Innere Medizin, Abteilung Pneumologie,

Rämistrasse 100, 8091 Zürich,

tél. 0125538 28, fax 0125544 51,

e-mail: konrad.bloch@dim.usz.ch

Pneumologie pédiatrique

PD Dr. Jürg Hammer,

Kinderspital Basel, Pneumologie,

Römergasse 8, 4005 Basel,

tél. 06168565 65, fax 06168550 59,

e-mail: juerg.hammer@unibas.ch

Veuillez commander le catalogue des branches d'examen au secrétariat de la Société Suisse de Pneumologie (SSP), Südbahnhofstrasse 14c, Case postale 49, 3000 Berne 17, tél 03137820 30, fax 03137820 31, e-mail: k.lehmann@lung.ch 